\title{
Vertical antiresonant reflecting optical waveguide coupler for three-dimensional optical interconnects: optimum design for large tolerance, high coupling efficiency, and short coupling length
}

\author{
Takashi Sekimoto, Shigenori Ikuta, Wugen Pan, Sai Tak Chu, and Yasuo Kokubun
}

\begin{abstract}
A compact antiresonant-reflecting-optical-waveguide- (ARROW-) type vertical coupler for threedimensional optical interconnects was demonstrated. The coupler consists of stacked ARROW's channeled by the stripe lateral confinement structure, and each waveguide is completely separated by a thin metal film in the separation region. In the coupling region the intermediate cladding of a previous coupler was made of the same material as that of the first cladding or the core. However, we had to overcome the problem that both the high coupling efficiency and the large fabrication tolerance cannot be achieved simultaneously. Thus we incorporated an intermediate cladding made of a material different from that of the core and the first cladding. The refractive index and the thickness of the intermediate cladding were optimally designed to achieve large fabrication tolerance and a short coupling length with a high coupling efficiency. The coupling length was reduced from 4.1 to $0.8 \mathrm{~mm}$, and a high coupling efficiency of $96 \%$ was experimentally demonstrated. (C) 2000 Optical Society of America

OCIS codes: $230.3120,230.7380,250.5300$.
\end{abstract}

\section{Introduction}

Integrated photonic circuits require a large chip area, owing to the restriction on the curvature of bends and branches. This is an obstacle to the dense integration of photonic circuits. The stacked configuration in photonic circuits is advantageous for the dense integration and large flexibility of layout design.

Since antiresonant reflecting optical waveguides (ARROW's) ${ }^{1}$ can be remotely coupled because of their nondecaying field profile inside the intermediate cladding, they are suitable for such stacked interconnects, and some stacked ARROW devices have been reported $^{2-6}$ However, these devices are predominantly planar devices.

T. Sekimoto, S. Ikuta, and Y. Kokubun (kokubun@dnj.ynu.ac.jp) are with the Department of Electrical and Computer Engineering, Faculty of Engineering, Yokohama National University, 79-5 Tokiwadai, Hodogayaku, Yokohama 240-8501, Japan. W. Pan, S. T. Chu, and Y. Kokubun are with the Kanagawa Academy of Science and Technology, 3-2-1 Sakato, Takatsuku, Kawasaki 213-0012, Japan.

Received 14 June 1999; revised manuscript received 18 October 1999.

0003-6935/00/030426-05\$15.00/0

(C) 2000 Optical Society of America
Some of the current authors previously proposed what are believed to be a new channeled structure of ARROW and a new vertical coupler that uses the structure $^{7-11}$ Because the channeled ARROW structure called stripe lateral confinement (SLC) can keep its top surface flat, other waveguides can be stacked on it. In addition, the stacked ARROW's can be completely isolated by a thin metal layer with minimal loss, and the vertical coupling structure can be easily formed by elimination of the material layer. ${ }^{7}$ However, the fabrication tolerance of the core thickness in the ARROW vertical coupler was very small (less than $\pm 1.4 \%$ for $80 \%$ coupling efficiency), and the coupling length was typically several millimeters. Therefore two types of improved vertical coupler were proposed. One is an improved vertical coupler that uses a thin intermediate cladding.8,9 This structure, however, cannot be stacked to more than three layers because of the lack of a metal separation layer. The other is an improved vertical coupler using a strong coupling structure. ${ }^{10}$ Although this type can be stacked to many layers, the maximum coupling efficiency is not high enough. In this paper the authors propose and demonstrate an optimized strong coupling structure of an ARROW vertical coupler and realize a short coupling length and a high coupling efficiency. 


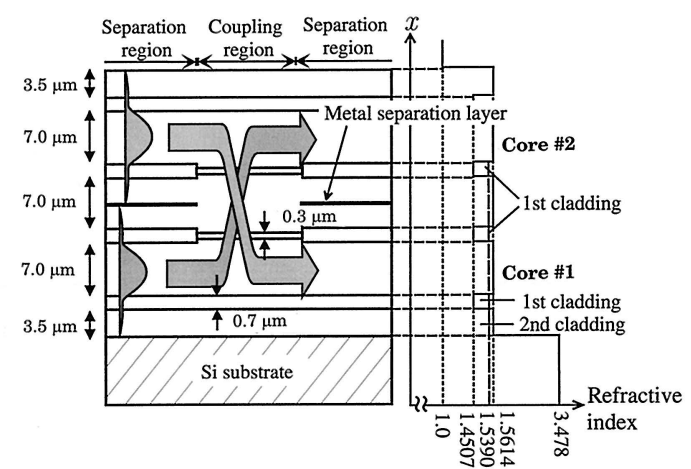

Fig. 1. Cross-sectional structure of stacked ARROW vertical coupler.

\section{Theory and Design}

Figure 1 shows the configuration of an ARROW-type vertical coupler with an optimized intermediate cladding layer in the coupler region. The core and the lower cladding are, respectively, $\mathrm{TiO}_{2} / \mathrm{Ta}_{2} \mathrm{O}_{5} / \mathrm{SiO}_{2}$ (8:4.7:87.3 mol. \%, $n=1.5614 @ \lambda=1.55 \mu \mathrm{m})$ and $\mathrm{SiO}_{2}(n=1.4507 @ \lambda=1.55 \mu \mathrm{m})$ formed on a Si substrate. In this structure the refractive index and the thickness of the intermediate cladding layer are optimized in the coupling region such that the difference of propagation constant between adjacent modes in the coupling region can be controlled to maintain high coupling efficiency. For the material of the intermediate cladding layer we adopted $\mathrm{Ta}_{2} \mathrm{O}_{5} / \mathrm{SiO}_{2}$ (6.5:93.5 mol. \%, $n=1.5390$ at $\lambda=1.55 \mu \mathrm{m})$, and the thickness is $0.3 \mu \mathrm{m}$.

When the spacing of propagation constants between adjacent modes in the coupling region is equal or odd multiples of the difference of the propagation constant between zeroth- and first-order modes, the coupling efficiency is close to $100 \%$. In the completely strong coupling structure ${ }^{10}$ in which the refractive index of the intermediate cladding is the same as that of the core, the maximum coupling efficiency is $\sim 63 \%$ because of the unequal spacing of propagation constants among guided modes in the coupling region.

Since the position of nodes (nulls) and peaks of the mode field is different for each mode order, the effect of intermediate cladding is different for each mode as shown in Fig. 2. Thus we can control the difference of the propagation constant by regulating the refractive index and the thickness of the intermediate clad-

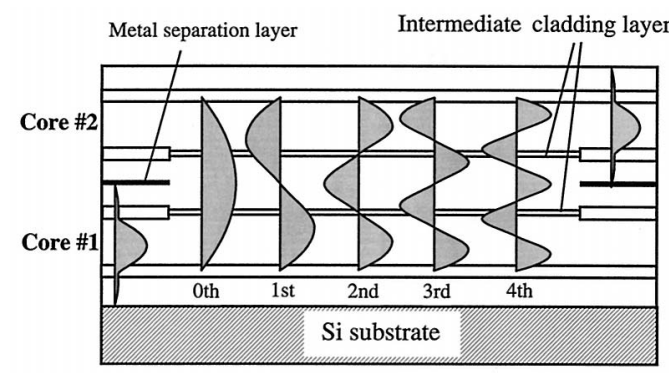

Fig. 2. Effect of intermediate cladding on the modal field and propagation constant of each mode.

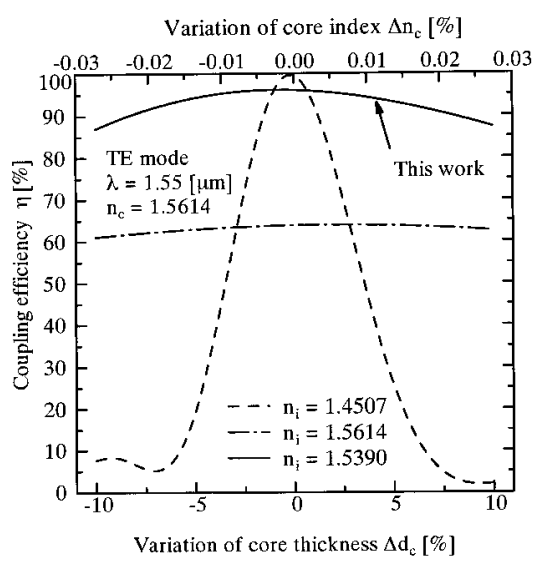

Fig. 3. Calculated fabrication tolerance of core thickness and index.

ding. As a result, high coupling efficiency, short coupling length, and large tolerance can be achieved simultaneously.

Since the coupling in this structure is stronger than that of the original vertical coupler structure, ${ }^{7}$ this structure can improve the problems of small fabrication tolerance and long coupling length. In addition, the refractive index of the intermediate cladding in the coupling region is slightly lower than that of the core, unlike the completely strong coupling structure, ${ }^{8-10}$ and a high coupling efficiency can be expected. In the separation region the intermediate cladding layer is $\mathrm{SiO}_{2}$, which has a low refractive index, so that the field is strongly confined in the core, and the propagation loss is kept small.

In this study we analyzed the fabrication tolerance of core thickness against the refractive index and the thickness of the intermediate cladding. In this analysis we used the two-dimensional eigenmode expansion method. The incident field was expanded in terms of zeroth- to fourth-order modes, because $99.2 \%$ of incident light power is expanded into these five modes. The calculated result is shown in Fig. 3. When the refractive index of the intermediate cladding is increased from a low value (1.4507) to the same refractive index as that of the core (1.5614), the fabri-

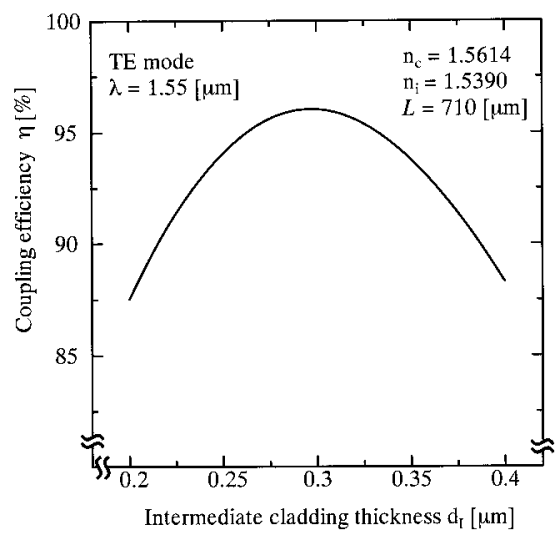

Fig. 4. Calculated fabrication tolerance of intermediate cladding thickness. 


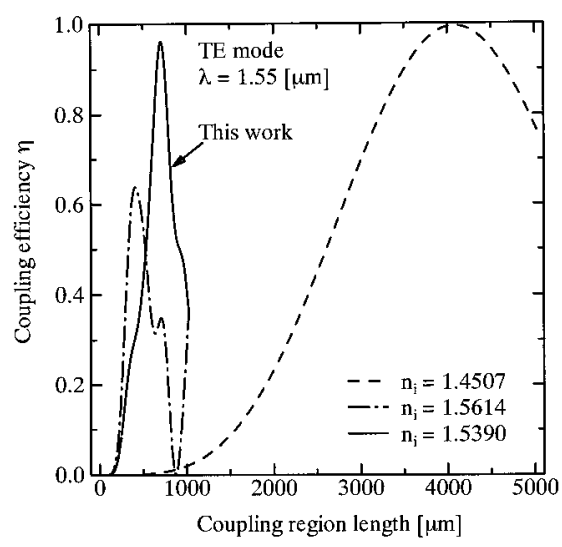

Fig. 5. Calculated coupling efficiency versus length of coupling region.

cation tolerance is improved drastically. However, the maximum coupling efficiency is as low as $63 \%$.

To improve the maximum coupling efficiency, a refractive index (1.5390) of intermediate cladding that is moderately lower than that of the core was adopted. The thickness is $0.3 \mu \mathrm{m}$. In the new configuration the maximum coupling efficiency shown by the solid curve in Fig. 3 is improved to $96 \%$, and the tolerance of the core thickness required for a higher than $90 \%$ coupling efficiency is improved to $\pm 8 \%$, which is much larger than the $\pm 1.4 \%$ of the original coupler structure.

Figure 4 shows the fabrication tolerance for the thickness of the intermediate cladding. The refractive indices of individual layers are the same as shown in Fig. 1. The coupler length $L$ was assumed to be $710 \mu \mathrm{m}$. The intermediate cladding thickness required for the coupling efficiency of higher than $90 \%$ ranges from 2.2 to $3.8 \mu \mathrm{m}$, which is wide enough for practical fabrication.
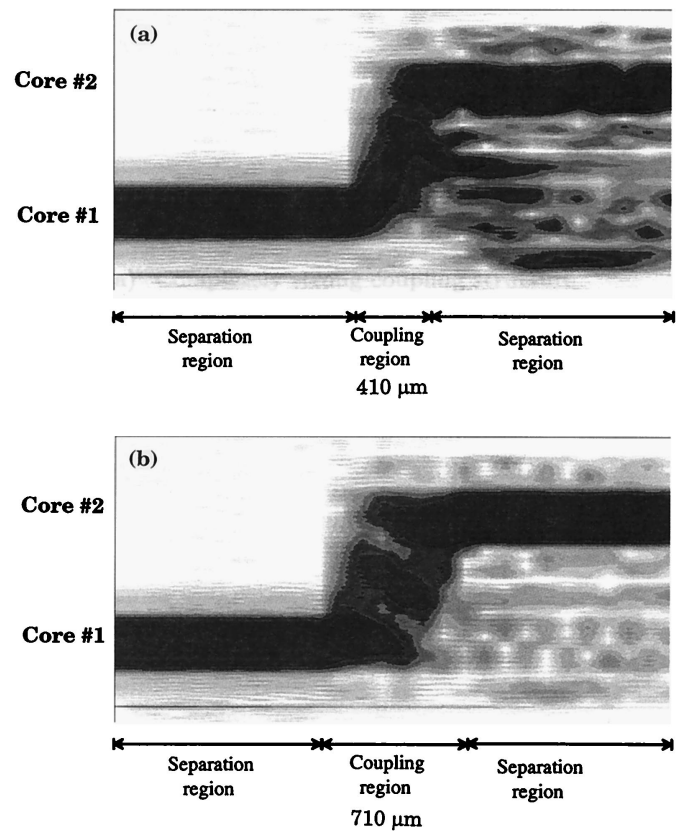

Fig. 6. Result of analysis by beam propagation method. (a) Completely strong coupling structure, (b) this paper.

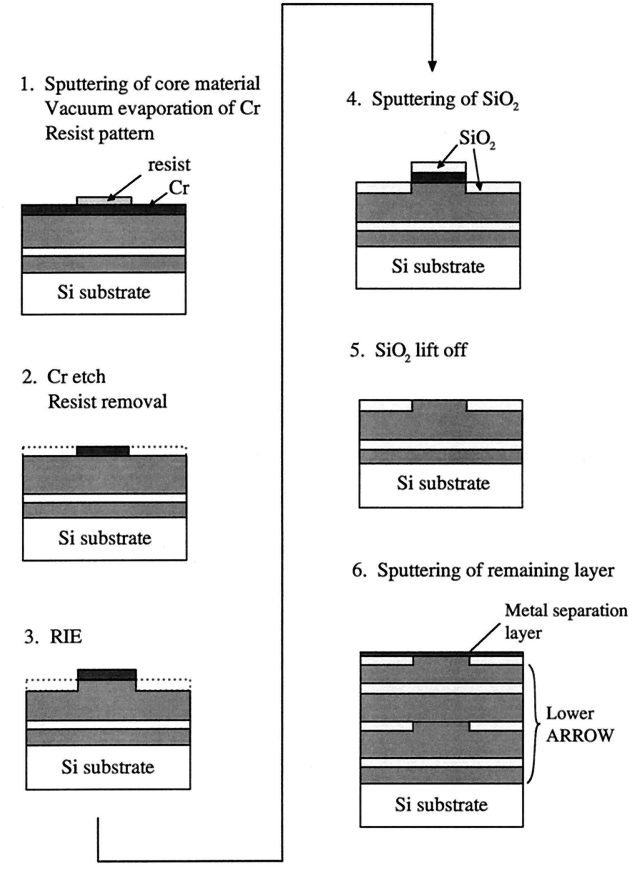

Fig. 7. Lift-off process used for the planarization of waveguide surface.

We also analyzed the coupling length, and the calculated results are shown in Fig. 5. It is seen that the coupling length is shortened from $4.1 \mathrm{~mm}$ of the previous structure ${ }^{7}$ to $\sim 750 \mu \mathrm{m}$. However, the intermediate cladding acts as the first cladding of the ARROW in the separation region. Thus the radiation loss in the separation region increases to several decibels/centimeter when the index of the intermediate cladding increases. Therefore the refractive index of the intermediate cladding must have a low value in the separation region and have an optimized value in the coupling region.

We analyzed the coupling characteristics by a twodimensional beam-propagation method. The thickness and the index of each layer are assumed to be the same as shown in Fig. 1. The calculated results are shown in Fig. 6. The light is incident on the lower left-hand waveguide and is intended to be coupled to the upper right-hand waveguide. In the completely strong coupling structure the light incident on the lower waveguide is not coupled completely to the upper waveguide, and the residual light is emitted from the lower waveguide, as shown in Fig. 6(a). However, in the structure of this study, the incident light is strongly coupled to the upper waveguide, as shown in Fig. 6(b).

\section{Fabrication and Measurement}

The thicknesses of the $\mathrm{TiO}_{2} / \mathrm{Ta}_{2} \mathrm{O}_{5} / \mathrm{SiO}_{2}$ (8:4.7:87.3 mol. \%) core; the $\mathrm{SiO}_{2}$ first cladding, which acts as the intermediate cladding in the separation region; and the SLC layer are 7.0, 0.7, and $0.5 \mu \mathrm{m}$, respectively. The thickness of the intermediate cladding in the coupling region, which is made of $\mathrm{Ta}_{2} \mathrm{O}_{5} / \mathrm{SiO}_{2}$ (6.5:93.5 mol. \%), is $0.3 \mu \mathrm{m}$. These materials were deposited 

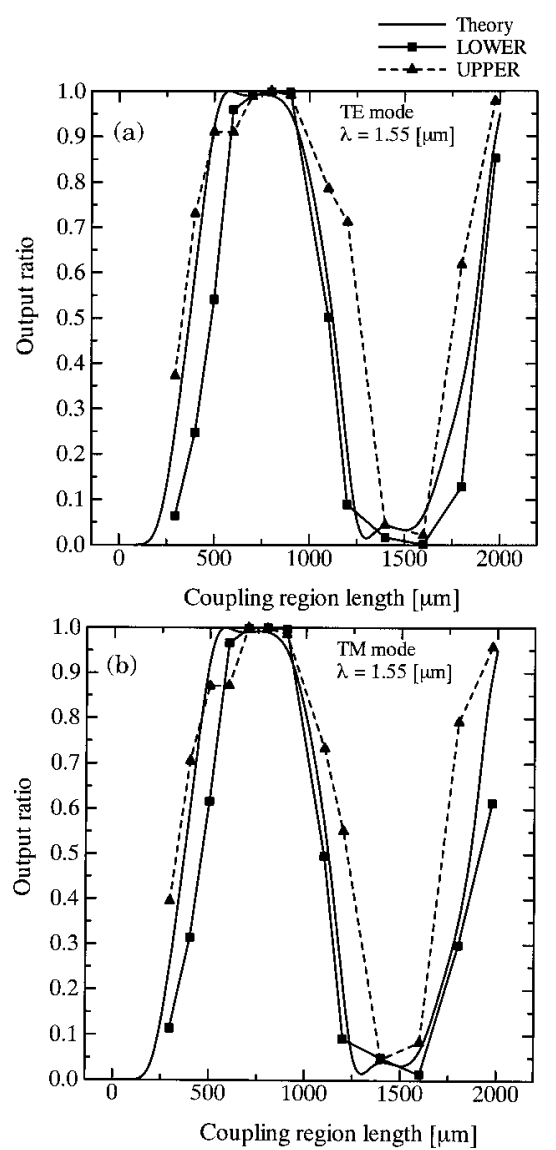

Fig. 8. Measured output ratio versus length of coupling region. (a) TE mode, (b) TM mode

by a rf magnetron sputtering technique by use of a gas mixture of $\mathrm{Ar}$ and $\mathrm{O}_{2}$ (1:3 volume ratio) with a rf power of $5.65 \mathrm{~W} / \mathrm{cm}^{2}$ and a gas pressure of $0.67 \mathrm{~Pa}$. The deposition rate was $1.5 \mu \mathrm{m} / \mathrm{h}$. When the channeled structure was formed, these layers were etched down by a reactive ion etching technique that uses $\mathrm{CF}_{4}$ with a rf power of $1.13 \mathrm{~W} / \mathrm{cm}^{2}$ and a gas pressure of $0.31 \mathrm{~Pa}$. The etching rate was $2.0 \mu \mathrm{m} / \mathrm{h}$ for $\mathrm{SiO}_{2}$ and $1.9 \mu \mathrm{m} / \mathrm{h}$ for core material. We used $\mathrm{Cr}$ as the mask for the etching of the core and the cladding materials.

We developed a lift-off process using the etching of the $\mathrm{Cr}$ layer and the lower waveguide fabrication process to realize the flat surface of the waveguide as

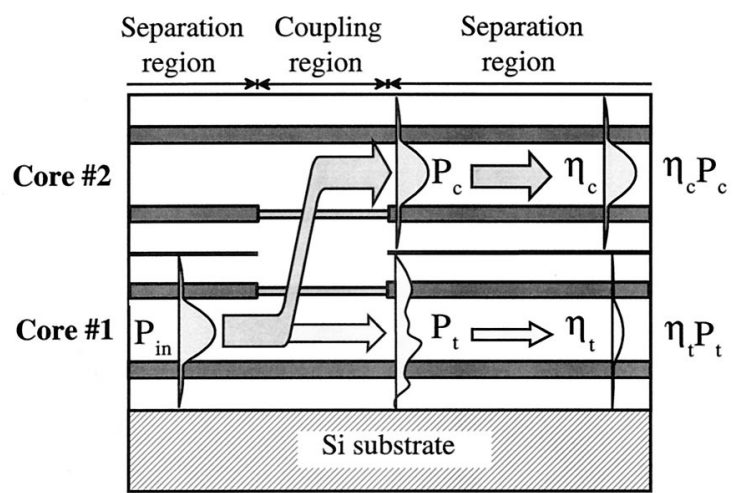

Fig. 9. Definition of output powers and coupling efficiencies.

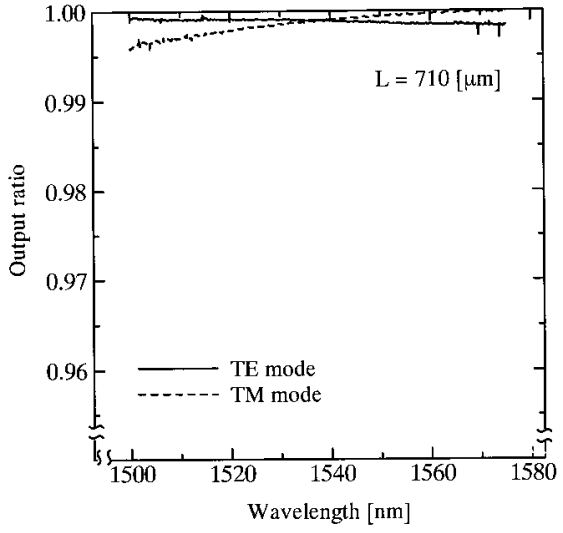

Fig. 10. Wavelength dependence of output power ratio.

shown in Fig. 7. First, the lower half of the ARROW structure is deposited by a rf magnetron sputtering technique, and a thin (15-nm) Cr layer is formed as shown in the first step of Fig. 7. After patterning the Cr mask with the photolithography and the wet etching as shown in the second step of Fig. 7, we etched down the core material $\left(\mathrm{TiO}_{2} / \mathrm{Ta}_{2} \mathrm{O}_{5} / \mathrm{SiO}_{2}\right)$ with a reactive ion etching technique as shown in the third step of Fig. 7. Next, the $\mathrm{SiO}_{2}$ layer on the $\mathrm{Cr}$ mask is lifted off by wet etching of $\mathrm{Cr}$, as shown in the fourth and the fifth steps of Fig. 7. Then a flat surface is obtained. The remaining upper half of the waveguide is deposited, and the SLC-ARROW is finished. Repeating this lift-off process, we obtain the stacked waveguide structure.

The upper and the lower waveguides were formed to be a channeled structure that uses the SLC structure, ${ }^{11}$ where the cross-sectional structure is shown in Fig. 11. In the separation region, a very thin metal layer (50-nm Cr layer) was inserted between the upper and the lower waveguides. This metal separation layer is essential for the threedimensional interconnects.

We measured the ratio of the output power of the coupled light to the whole output power, which is the sum of output powers from lower and upper waveguides. This ratio is called the output ratio. Figure 8(a) shows the measured results of the output

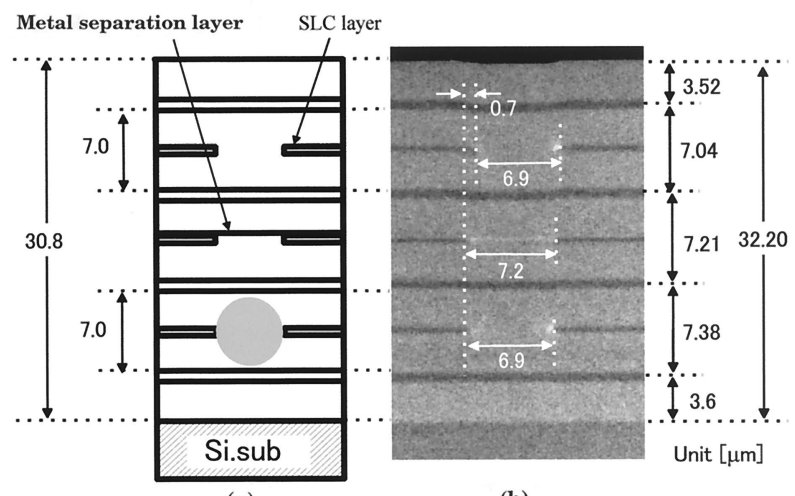

(a)

(b)

Fig. 11. Cross-sectional view of the coupler. The unit of all numbers is micrometers. (a) Designed structure, (b) scanning electron microscope image of the device. 


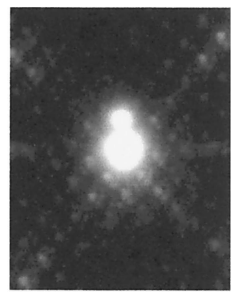

$\mathrm{L}=295 \mu \mathrm{m}$

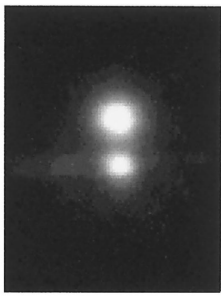

$500 \mu \mathrm{m}$

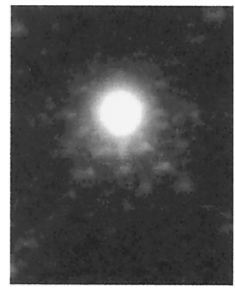

$800 \mu \mathrm{m}$

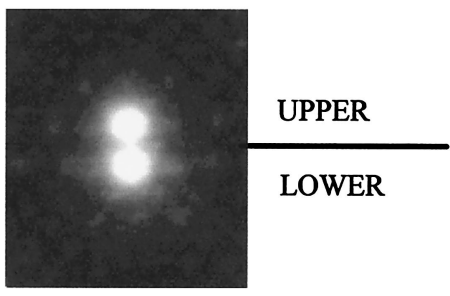

$1100 \mu \mathrm{m}$

Fig. 12. Observed near-field images at different lengths of coupling region.

ratio for the TE mode and Fig. 8(b) for the TM mode. The squares and triangles in Fig. 8 denote the measured output ratios when the light was incident on the lower and the upper waveguides, respectively. The output ratio was obtained to be larger than 0.99 at $L=800 \mu \mathrm{m}$ for both TE and TM modes. Since the theoretical value of the maximum output ratio is $\sim 0.99$ at the coupling length of $750 \mu \mathrm{m}$, the measured result coincides with the theory.

The output ratio is not related directly to the coupling efficiency. The propagation loss was evaluated to be $0.2-0.5 \mathrm{~dB} / \mathrm{cm}$ with the cutback method, and this value does not affect the output ratio. The output ratio $R$ is expressed by

$$
R=\frac{\eta_{c} P_{c}}{\eta_{c} P_{c}+\eta_{t} P_{t}},
$$

where $P_{c}$ is the light power directed to the objective output port; $P_{t}$ is the uncoupled light power passed through the same waveguide as the input port; and $\eta_{c}$ and $\eta_{t}$ are the coupling efficiencies of light to the objective and throughports, respectively, as shown in Fig. 9. Since the field profile of the light directed to the objective port is close to the mode field of the output waveguide, $\eta_{c} \simeq 1.0$. However, the uncoupled light is hard to couple to the throughport, because the field profile is not close to that of the eigenmode. Thus, when the coupling efficiency is $96 \%, 4 \%$ of incident power is directed to the throughport. Since a small amount of this power $(\sim 10 \%)$ is guided in the throughport, however, the output ratio reaches 0.996 .

The wavelength dependence of the output power ratio was also measured, and the results for the TE and the TM modes are shown in Fig. 10. The dependence was confirmed to be less than 1\% for both TE and TM modes in the 1.50-1.58- $\mu \mathrm{m}$ wavelength range.

Figure 11 shows the cross-sectional scanning electron microscope view of the coupler. The left-hand part is the designed structure, and the right-hand one is the observed scanning electron microscope photo of the device. It is seen from these figures that the core thickness of upper and lower waveguides differ by $\sim 5 \%$. In spite of this fabrication error, a high output ratio was successfully obtained, owing to the optimized strong coupling structure.

The near-field images of the output at different lengths of the coupling region are shown in Fig. 12. It can be seen from these figures that the vertical coupling was successfully observed.

\section{Conclusion}

We have demonstrated a new, to our knowledge, configuration of an ARROW-type vertical coupler, using the optimized intermediate cladding in the coupler region. A high output ratio greater than 0.99 at $L=$ $800 \mu \mathrm{m}$ and low polarization and wavelength dependencies were successfully demonstrated.

The authors thank M. Maeda of the Hitachi Company, Ltd. for supplying the laser diode. At the Kanagawa Academy of Science and Technology, this project ended in March 1999.

\section{References}

1. M. A. Duguay, Y. Kokubun, T. L. Koch, and L. Pfeiffer, "Antiresonant reflecting optical waveguides in $\mathrm{SiO}_{2}-\mathrm{Si}$ multi-layer structures," Appl. Phys. Lett. 49, 13-15 (1986).

2. B. Pezeshki, F. F. Tong, J. A. Kash, and D. W. Kisker, "Vertical cavity devices as wavelength selective waveguides," J. Lightwave Technol. 12, 1791-1801 (1994).

3. T. Baba, Y. Kokubun, T. Sasaki, and K. Iga, "Loss reduction of an ARROW waveguide in shorter wavelength and its stack configuration," J. Lightwave Technol. 6, 1440-1445 (1988).

4. M. Mann, U. Trustshel, C. Wächter, L. Leine, and F. Lederer, "Directional coupler based on an antiresonant refracting optical waveguide," Opt. Lett. 16, 805-807 (1991).

5. B. Pezeshki, J. A. Kash, D. W. Kisker, and F. Tong, "Multiple wavelength light source using an asymmetric waveguide coupler," Appl. Phys. Lett. 65, 138-140 (1994).

6. U. Trutshel, F. Ouellette, V. Delisle, M. A. Duguay, G. Fogarty, and F. Lederer, "Polarization splitter based on antiresonant reflecting optical waveguide," J. Lightwave Technol. 13, 239-243 (1995).

7. S. Asakawa, Y. Kokubun, M. Ohyama, and T. Baba, "Threedimensional optical interconnects by stacked ARROW waveguides," Electron. Lett. 29, 1485-1486 (1993).

8. M. Ohyama, Y. Kokubun, and E. Ohta, "Compact threedimensional optical interconnects with large tolerance by stacked ARROW-type waveguides,” Electron. Lett. 30, 951-952 (1994).

9. S. T. Chu, Y. Kokubun, and M. Miura, "Versatile stacked ARROW crossconnects for three-dimensional optical interconnects," Electron. Lett. 31, 33-35 (1994).

10. S. Ikuta, S. Kubota, W. Pan, S. T. Chu, and Y. Kokubun, "Stacked ARROW vertical coupler with large tolerance and short coupling length for three-dimensional interconnects," Electron. Lett. 34, 1851-1852 (1998).

11. T. Baba, Y. Kokubun, and Y. Mera, "Novel 3-D ARROW by thin film patterning: stripe lateral confinement of ARROW," in Integrated Guided Wave Optics, Vol. 4 of 1989 OSA Technical Digest Series (Optical Society of America, Washington, D.C., 1989), paper TuBB5. 